\section{A FORTRAN IV program for Rao's test of uniformity in circular distributions}

\author{
PAUL V. HAMILTON \\ Department of Biological Science, Florida State University \\ Tallahassee, Florida 32306
}

Circular distributions are often statistically analyzed using a null hypothesis of a uniform distribution and an alternate hypothesis of a unimodal distribution (e.g., the Rayleigh test described by Batschelet, 1972). However, bimodal or multimodal distributions are frequently encountered in behavioral studies (e.g., studies of orientation to polarized light or wave surge, activity rhythm studies), and unimodal tests will often fail to reject the null hypothesis of a uniform distribution even though distinct modes are apparent. Rao proposed a test which is powerful for both unimodal and multimodal alternatives. Examples of this test's application are given by Batschelet (1972) and Rao and Sengupta (1972). Although theoretically simple, Rao's test can be computationally tedious for even medium-sized data sets.

Computer Method. All computations are completed and all output is generated by a single subroutine. Containment of all procedures for this test in a subroutine permits its use with other appropriate subroutines in a calling program for rapid and complete analysis of a data set. The computations for Ran's test are described in Rao and Sengupta (1972).

This program was developed with the support of the Florida State University Psychobiology Research and Computing Centers. Comments by L. Bloom and D. Howard are appreciated. Present address: Faculty of Biology, University of West Florida, Pensacola, Florida 32504.
Input. Data values may be in degrees, radians, or units of time (hours, days, months), with the user specifying the maximum possible value in each case (e.g., $360 \mathrm{deg}$, $24.00 \mathrm{~h}$ ). Comments in the program source listing explain the simple modifications required to increase the maximum permissible number of data values from its present limit of 99.

Output. (1) An optional table listing the input data, the ordered input data, the arc lengths, and the deviations of the arc lengths from the mean value (all variables are in the units of the data values). (2) The sample size (n). (3) The mean arc length (in the units of the data values). (4) The test statistic (U) for the hypothesis that the sample distribution comes from a uniform population. The test statistic is in units of degrees, regardless of the units of the data values, to conform with published tables of critical values [see_Batschelet (1972) for the most extensive table].

Computer and Language. The program is written in FORTRAN IV and was tested on the Florida State University CYBER 70 computer.

Availability. Copies of the program listing and documentation can be obtained without charge from Paul V. Hamilton, Faculty of Biology, University of West Florida, Pensacola, Florida 32504.

\section{REFERENCES}

Batschelet, E. Recent statistical methods for orientation data. In S. R. Galler, K. Schmidt-Koenig, G. J. Jacobs, \& R. E. Belleville (Eds.), Animal orientation and navigation (Stock No. 3300-0392). Washington, D.C: U.S. Government Printing Office, 1972. Pp. 61-91.

RAo, J. S. \& SEngupta, S. Mathematical techniques for paleocurrent analysis: Treatment of directional data. Mathematical Geology, 1972, 4, 235-248. 